\title{
GOURSAT'S THEOREM AND HOMOLOGICAL ALGEBRA
}

\author{
Joachim Lambek \\ (received February 7, 1964)
}

This expository note consists of two parts: In the first we present a homological formulation of Goursat' s theorem. In the second we indicate how some rudimentary concepts of homological algebra can be introduced with the help of this form of Goursat's theorem. The second part is addressed to those readers with an algebraic background who wish to be initiated into homological algebra as painlessly as possible. The ideas developed here are close to the spirit of [5, Chapter II, $\S 6]$, where further references may be found. In fact, the se ideas are very much in the air, and any originality in the present note is purely coincidental.

\section{PART I}

Before stating Goursat's theorem, some preliminary definitions are in order. If $A$ and $B$ are sets, a binary relation between $A$ and $B$ is a triple $\rho=(R, A, \bar{B})$, where $R$ is a subset of the cartesian product $A \times B$, called the graph of $\rho$. Now let $A$ and $B$ be groups; then $\rho$ is called a homomorphic relation provided $R$ is a subgroup of $A \times B$. Well-known examples of homomorphic relations between $A$ and B are:

(a) homomorphisms of $A$ into $B$,

(b) inverses of homomorphisms of $B$ into $A$,

(c) congruence relations on $A$ (in case $A=B$ ).

We write $a \rho b$ to mean $(a, b) \in R$.

GOURSAT'S THEOREM. If $\rho$ is any homomorphic relation between groups $A$ and $B$ then the following factorgroups are isomorphic:

Canad. Math. Bull. vol. 7, no. 4, October 1964 


$$
\frac{\left\{a \in A \mid \Xi_{b \in B} a \rho b\right\}}{\{a \in A \mid a \rho 0\}} \cong \frac{\left\{b \in B \mid \Xi_{a \in A} a \rho b\right\}}{\{b \in B \mid 0 \rho b\}}
$$

(The neutral element of a group has been designated as 0 , since we ultimately have abelian groups in mind.)

Historical note. Essentially this result was first published in 1889 (see [3]). It has been rediscovered many times since, for example by Shoda (see [6]). A recent discussion of this theorem may be found in [4] where it is also shown how the Jordan-Hölder-Schreier Theorem may be deduced from it. (It furthermore follows from [4] that a slightly modified form of Goursat's Theorem remains valid in any class of algebraic systems (i.e. sets with operations) provided among the se operations there is a ternary operation $f(x, y, z)$ such that identically

$$
f(x, y, y)=x, \quad f(y, y, z)=z .
$$

The modification consists in replacing normal subgroups by congruence relations.)

We now present a homological formulation of Goursat's Theorem, which is illustrated by the following diagram.

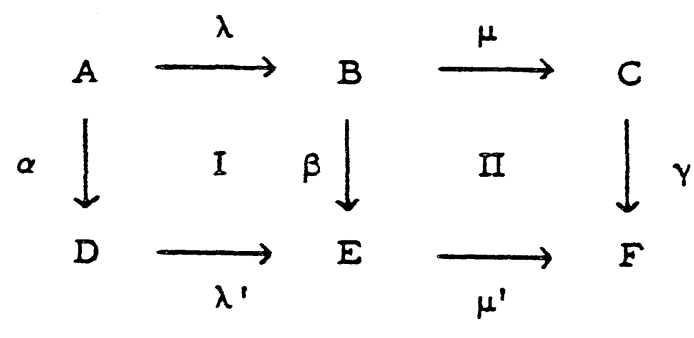

THEOREM. Let $\lambda, \mu, \lambda^{\prime}, \mu^{\prime}, \alpha, \beta, \gamma$ be given homomorphisms of groups such that

$$
\operatorname{Im} \lambda=\operatorname{Ker} \mu, \quad \operatorname{Im} \lambda^{\prime}=\operatorname{Ker} \mu^{\prime},
$$

and

$$
\beta \circ \lambda=\lambda^{\prime} \circ \alpha, \quad \gamma \circ \mu=\mu^{\prime} \circ \beta \text {; }
$$


then

$$
\frac{\operatorname{Im} \beta \cap \operatorname{Im} \lambda^{\prime}}{\operatorname{Im}(\beta \circ \lambda)} \cong \frac{\operatorname{Ker}(\gamma \circ \mu)}{\operatorname{Ker} \beta+\operatorname{Ker} \mu}
$$

CONMENTS. The group operation has been written + , without assuming commutativity. Since Ker $\beta$ and Ker $\mu$ are normal subgroups of $B$, their sum is the normal subgroup generated by them. Also $\operatorname{Im}(\beta \circ \lambda)=\beta(\operatorname{Ker} \mu)$ is a normal subgroup of $\operatorname{Im} \beta=\beta B$.

One usually renders (1) by saying that the rows of the diagram are exact.

One usually renders (2) by saying that the squares I and II of the diagram are commutative.

We suggest that (3) be read thus:

The image ratio of $I$ is isomorphic with the kernel ratio of II. We write $i(I) \cong k$ (II).

Proof. For $b \in B$ and $e \in E$ write $b \rho e$ to mean that there exists $b^{\prime} \in B$ such that

$$
\mu b=\mu b^{\prime} \text { and } \beta b^{\prime}=e \text { and } \mu^{\prime} e=0 \text {. }
$$

The reader will have no difficulty in verifying that $\rho$ is a homomorphic relation between $B$ and $E$. Now compute:

$$
\begin{aligned}
& \text { (i) } \exists_{e \in E^{b} \rho e} \Leftrightarrow \exists_{b^{\prime} \in B^{\mu b}=\mu b^{\prime}} \text { and } \mu^{\prime}\left(\beta b^{\prime}\right)=0 \\
& \Leftrightarrow \Xi_{b^{\prime} \in B^{\mu b}=\mu b^{\prime}} \text { and } \gamma(\mu b)=0 \\
& \Leftrightarrow \quad b \in \operatorname{Ker}(\gamma \circ \mu), \\
& \text { (ii) } b \rho 0 \quad \Leftrightarrow \Xi_{b^{\prime} \in B^{\mu}}\left(b-b^{\prime}\right)=0 \text { and } \beta b^{\prime}=0 \text {. } \\
& \Leftrightarrow \quad b \in \operatorname{Ker} \mu+\operatorname{Ker} \beta .
\end{aligned}
$$


(iii) $\Xi_{b \in B} b \rho e \Leftrightarrow \Xi_{b^{\prime} \in B} \beta b^{\prime}=e$ and $\mu^{\prime} e=0$

$$
\Leftrightarrow \quad \quad \quad \in \operatorname{Im} \beta \cap \operatorname{Im} \lambda^{\prime} \text {. }
$$

(iv) $0 \rho \mathrm{e}$

$$
\begin{aligned}
& \Leftrightarrow I_{b^{\prime} \in B^{\prime}} 0=\mu b^{\prime} \text { and } \beta b^{\prime}=e \text { and } \mu^{\prime} e=0 \\
& \Leftrightarrow \quad e \in \beta(\operatorname{Ker} \mu) \text { and } \mu^{\prime} e=0 \\
& \Leftrightarrow \quad \quad \quad \in \in \operatorname{Im}(\beta \circ \lambda) .
\end{aligned}
$$

since $\mu^{\prime}(\beta \operatorname{Ker} \mu)=\gamma(\mu \operatorname{Ker} \mu)=0$.

Applying Goursat's Theorem to the relation $\rho$ defined above, we immediately obtain (3).

We shall now show that conversely Goursat's Thoerem may be deduced from the above result.

Let $A$ and $B$ be given groups, $R$ a subgroup of $A \times B$. Then $p=(R, A, B)$ is a homomorphic relation between $A$ and B. Put

$$
\begin{aligned}
& C=\left\{a \in A \mid I_{b \in B} a \rho b\right\}, \\
& D=\{a \in A \mid a \rho 0\}, \\
& C^{\prime}=\left\{b \in B \mid I_{a \in A} a \rho b\right\}, \\
& D^{\prime}=\{b \in B \mid 0 \rho b\},
\end{aligned}
$$

and consider the following diagram:

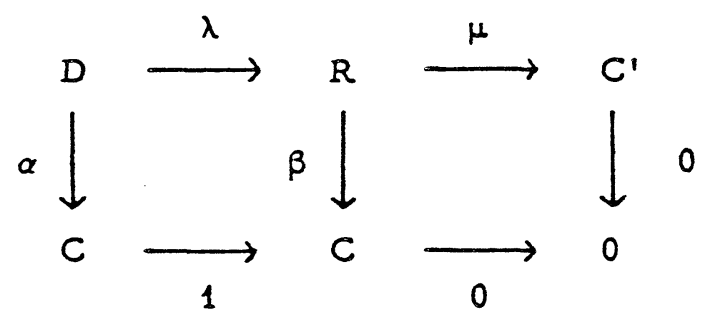


Here $\alpha$ is the inclusion map, $\lambda d=(d, 0) \in R$, for any $d \in D$, $\beta\left(c, c^{\prime}\right)=c \in A$, for any $\left(c, c^{\prime}\right) \in R$, $\mu\left(c, c^{\prime}\right)=c^{\prime} \in B$, for any $\left(c, c^{\prime}\right) \in R$.

We now verify (1) and (2); thus

$$
\begin{aligned}
\left(c, c^{\prime}\right) \in \operatorname{Im} \lambda & \Leftrightarrow c \in D \text { and } c^{\prime}=0 \\
& \Leftrightarrow\left(c, c^{\prime}\right) \in \operatorname{Ker} \mu,
\end{aligned}
$$

and the exactness of the bottom row is obvious. Furthermore

$$
(\beta \circ \lambda) d=\beta(d, 0)=d=\left(\begin{array}{lll}
1 \circ \alpha & 0
\end{array}\right) \text {, }
$$

and the commutativity of the second square is obvious. By our theorem we have (3). Now

$$
\begin{aligned}
& \operatorname{Im} \beta \cap \operatorname{Im} 1=C \cap C=C, \\
& \operatorname{Im}(\beta \circ \lambda)=D, \\
& \operatorname{Ker}(0 \circ \mu)=R, \\
& \operatorname{Ker} \beta+\operatorname{Ker} \mu=\left(0 \times D^{\prime}\right)+(D \times 0) \\
&=D \times D^{\prime} .
\end{aligned}
$$

Hence (3) asserts that

$$
C / D \cong R /\left(D \times D^{\prime}\right)
$$

Because of the symmetry of the right hand side of this isomorphism, we have

$$
C / D \cong C^{\prime} / D^{\prime}
$$

and this is Goursat's theorem.

As a byproduct of this proof we obtain the following supplementary result to Goursat's Theorem.

COROLLARY. Both factor-groups in Goursat's Theorem are isomorphic to $R /\{(a, b) \in R \mid a p 0$ and $0 \rho b\}$. 
Since it is not fashionable to have a proof without a result proved by it, we state the following

INFORMAL METATHEOREM. The above theorem is equivalent to Goursat's Theorem.

To give a precise meaning to this statement one must go into the axiomatics of categories, something which we shall not do here.

We illustrate the power of Goursat's Theorem in its homological form by giving a rapid construction of the so-called connecting homomorphism.

Consider the following diagram, in which all rows and columns are as sumed to be exact and all squares are as sumed to be commutative.

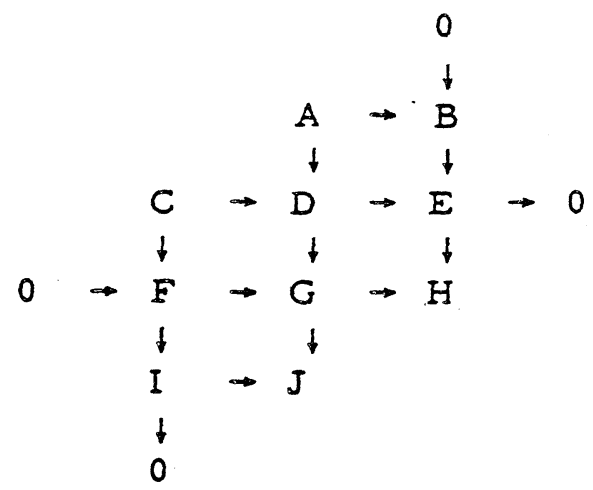

We want to show the existence of a homomorphism $B \rightarrow I$ so that the sequence

$$
\mathrm{A} \rightarrow \mathrm{B} \rightarrow \mathrm{I} \rightarrow \mathrm{J}
$$

is exact. Thus let $X=B / K$, where $K$ is the image of the homomorphism $A \rightarrow B$, and let $Y$ be the kernel of the homomorphism $I \rightarrow J$, then we want to show that $X \cong Y$. (Why is $K$ normal in $B$ ? If we identify $B$ with its image in $E, K$ is the image in $E$ of the image of $A \rightarrow D$, that is of the kernel of $D \rightarrow G$. Since the latter is normal in $D, K$ is actually normal in E.) 
We may enlarge part of our diagram as follows, so that again all rows and columns are exact and all squares are commutative.

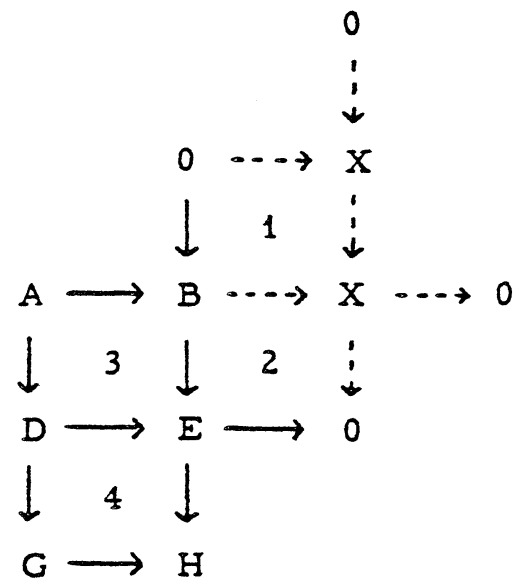

We now compute image and kernel ratios:

$$
x=i(1) \cong k(2) \cong i(3) \cong k(4) \text {. }
$$

Since the square labelled 4 was symmetrically situated in the original diagram, we also have $Y \cong k(4)$; hence $X \cong Y$, as was to be shown.

\section{PART II}

Given any exact sequence of right $\mathrm{R}$-modules

$$
\mathrm{O} \rightarrow \mathrm{A} \stackrel{\operatorname{m}}{\rightarrow} \mathrm{B} \rightarrow \mathrm{C} \rightarrow 0,
$$

it is well-known (e.g. [5], p. 148) that, for any left R-module $M$, the canonically associated sequence

$$
A \otimes_{R} \stackrel{k \otimes 1}{M} \rightarrow \otimes_{R} \otimes_{R} \stackrel{\pi \otimes 1}{\rightarrow}{ }^{M} \otimes_{R} M \rightarrow 0
$$

is also exact. Moreover $R^{M}$ is called flat if this latter exact sequence can be completed by sticking $0 \rightarrow$ in front. We assume 
as known that any iree module is flat [1, p. 28]. Henceforth we shall write $\otimes$ in place of $\otimes_{R}$.

Let $A_{R}$ and $R^{\prime}$ be given $R$-modules. We can find flat modules $F_{R}$ and $F^{\prime}$ and epimorphisms $\pi: F \rightarrow A$ and $\pi^{\prime}: F^{\prime} \rightarrow A^{\prime}$, for example by taking $F$ and $F^{\prime}$ to be free. Putting $K=\operatorname{Ker} \pi$ and $K^{\prime}=\operatorname{Ker} \pi^{\prime}$, we obtain exact sequences

$$
0 \rightarrow \mathrm{K} \stackrel{\kappa}{\rightarrow} \mathrm{F} \stackrel{\pi}{\rightarrow} \mathrm{A} \rightarrow 0, \quad 0 \rightarrow \mathrm{K}^{\prime} \stackrel{K^{\prime}}{\rightarrow} \mathrm{F}^{\prime} \stackrel{\pi^{\prime}}{\rightarrow} \mathrm{A}^{\prime} \rightarrow 0,
$$

where $x$ and $x^{\prime}$ are the inclusion mappings. Now let

$$
X=\operatorname{Ker}\left(\kappa \otimes 1_{A^{\prime}}\right), \quad X=\operatorname{Ker}\left(1_{A} \otimes \kappa^{\prime}\right)
$$

and consider the following diagram:

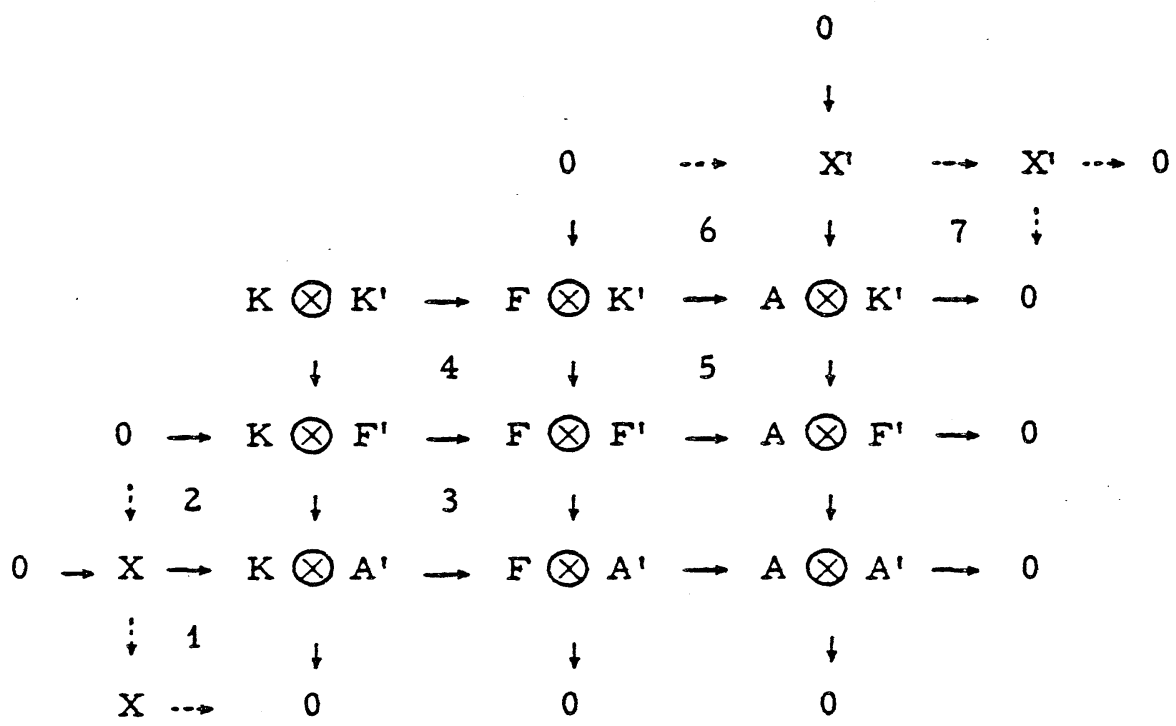

It follows from what has been said above that all rows and columns in this diagram are exact. In particular, the second undotted column is exact because $F$ is flat. Moreover, one readily sees that all squares in the diagram are commutative. For example, the compound mapping of $K \otimes K^{\prime}$ into $F \otimes F^{\prime}$ is 


$$
\left(1 \otimes K^{\prime}\right) \circ(\kappa \otimes 1)=\kappa \otimes K^{\prime}
$$

one way, and

$$
(K \otimes 1) \circ\left(1 \otimes K^{\prime}\right)=K \otimes K^{\prime}
$$

the other.

Applying Goursat's Theorem in its homological form, we have

$$
x=k(1) \cong i(2) \cong k(3) \cong i(4) \cong k(5) \cong i(6) \cong k(7)=X
$$

Retaining only $X, i(4)$ and $X^{\prime}$, we obtain the following wellknown result.

PROPOSITION. Let $0 \rightarrow \mathrm{K} \stackrel{\kappa}{\rightarrow} \mathrm{F} \stackrel{\pi}{\rightarrow} \mathrm{A} \rightarrow 0$ be an exact sequence of right $R$-modules with $F_{R}$ flat, and let

$$
0 \rightarrow K^{\prime} \stackrel{x^{\prime}}{\rightarrow} F^{\prime} \stackrel{\pi^{\prime}}{\rightarrow} A^{\prime} \rightarrow 0
$$

be an exact sequence of left $R$-modules with $R^{\prime}$ flat. Then $\operatorname{Ker}\left(k \otimes 1_{A^{\prime}}\right) \cong \frac{\operatorname{Im}\left(k \otimes 1_{F^{\prime}}\right) \cap \operatorname{Im}\left(1_{F} \otimes K^{\prime}\right)}{\operatorname{Im}\left(\kappa \otimes K^{\prime}\right)} \cong \operatorname{Ker}\left(1_{A} \otimes \kappa^{\prime}\right)$

Since $\operatorname{Ker}\left(\kappa \otimes{ }_{A^{\prime}}\right)$ does not depend on the sequence $0 \rightarrow K \rightarrow F \rightarrow A \rightarrow 0$ and $\operatorname{Ker}\left(1_{A} \otimes K^{\prime}\right)$ does not depend on the sequence $0 \rightarrow K^{\prime} \rightarrow F^{\prime} \rightarrow A^{\prime} \rightarrow 0$, both can depend only on $A$ and $A^{\prime}$. One writes Tor $\left(A, A^{\prime}\right)$ for this common value (common up to isomorphism that is), and calls it the torsion product of $A$ with $A^{\prime}$. (Actually, the usual definition of torsion products uses projective in place of flat modules.)

We shall desist from developing any properties of the torsion product here. Let us only point out that even when $F$ is not flat, the exact sequence

$$
0 \rightarrow \mathrm{K} \rightarrow \mathrm{F} \rightarrow \mathrm{A} \rightarrow 0
$$


gives rise to an exact sequence involving $\otimes$, to wit

$$
\begin{aligned}
\operatorname{Tor}\left(K, A^{\prime}\right) & \rightarrow \operatorname{Tor}\left(F, A^{\prime}\right) \rightarrow \operatorname{Tor}\left(A, A^{\prime}\right) \\
& \rightarrow K \otimes A^{\prime} \rightarrow F \otimes A^{\prime} \rightarrow A \otimes A^{\prime} \rightarrow 0 .
\end{aligned}
$$

This is shoun with the help of the connecting homomorphism of Part I.

Finally let it be mentioned that the functor Ext $(A, B)$, for right $R$-modules $A$ and $B$, may be introduced in an entirely analogous manner, by starting with exact sequences

$$
0 \rightarrow \mathrm{K} \rightarrow \mathrm{P} \rightarrow \mathrm{A} \rightarrow \mathrm{O}, \quad \mathrm{O} \rightarrow \mathrm{B} \rightarrow \mathrm{I} / \mathrm{B} \rightarrow 0,
$$

where $P$ is projective and $I$ is injective.

While the results of Part I are valid for non-abelian groups (and even more general systems, see [4]), in Part II we have confined attention to $R$-modules, thus to abelian groups. It is obvious though that Part II would remain valid for non-abelian groups (take $R$ to be the ring of integers), provided we had a non-abelian functor corresponding to $\otimes$. I am endebted to Basil Rattray for the observation that the most natural attempt to introduce a tensor product of two non-abelian groups leads to the usual tensor product of their abelianizations.

\section{REFERENCES}

1. N. Bourbaki, Éléments de Mathématique XXVII, Paris 1961.

2. H. Cartan and S. Eilenberg, Homological Algebra, Princeton 1956.

3. É. Goursat, Sur les substitutions orthogonales.... Ann. Sci. Éc. Norm. Sup. (3), 6(1889), 9-102.

4. J. Lambek, Goursat's Theorem and the Zas senhaus Lemma, Can. J. Math. 10(1958), 45-56. 
5. S. MacLane, Homology, New York 1963.

6. K. Shoda, Allgemeine Algebra, Osaka Math. J. 2 (1949), $182-225$.

\section{POSTSCRIPT}

Peter Hilton informs me that he has independently discovered the theorem of Part I.

The following additional references, concerning the last paragraph only, have been pointed out to the author:

I. Fleischer, A note on subdirect products, Acta Math. Acad. Sci. Hungar. 6 (1955), 463-465.

A. Frốhlich, Non-abelian homological algebra, Proc. London Math. Soc. 11 (1961), 239-75, and 12 (1962), 1-28, 739-68.

T. MacHenry, The tensor product and the 2 nd nilpotent product of groups, Math. Z. 73 (1960), 134-145.

, The tensor product of non-abelian groups and exact sequences, Arch. Math. 11((1960), 166-170.

\section{McGill University}




\section{NOTES ADDED IN PROOF}

1. Actually, Goursat's original theorem also asserted the following (in the notation of the theorem stated at the beginning of Part I): "Moreover $a \rho b$ if and only if the equivalence classes of $a$ and $b$ correspond under this isomorphism." While this additional fact is not used in the present paper, the historical note in Part I is really about this augmented theorem.

2. If two commutative squares are suitably attached to a third square so that all rows and columns are exact, one deduces that their image ratios are isomorphic. It has come to my attention that this isomorphism was used by B. Eckmann in his lectures some years ago.

3. Here is another, more direct proof of the two-square theorem:

$$
\begin{aligned}
\operatorname{Im} \beta \cap \operatorname{Im} \lambda^{\prime} & =\operatorname{Im} \beta \cap \operatorname{Ker} \mu^{\prime}=\left\{\beta b \mid \mu^{\prime} \beta b=0\right\} \\
& =\{\beta b \mid \gamma \mu b=0\}=\beta \operatorname{Ker}(\gamma \circ \mu) . \\
\operatorname{Im}(\beta \circ \lambda) & =\beta \operatorname{Im} \lambda=\beta \operatorname{Ker} \mu=\beta(\operatorname{Ker} \mu+\operatorname{Ker} \beta) .
\end{aligned}
$$

The result now follows from one of the classical isomorphism theorems of group theory. 